\title{
Características morfológicas da distribuição vascular cerebral de Sus scrofa Linnaeus (Mammalia, Artiodactyla)
}

\author{
Jurandyr de A. Câmara Filho 1, 2, Paulo O. Scherer ${ }^{1}$, Raphael R. Scherer ${ }^{1}$, Carlos M. C. de Menezes ${ }^{3}$ \\ 1 Programa de Pós-graduação em Biologia Animal, Instituto de Biologia Animal, Universidade Federal Rural do Rio de Janeiro. \\ 23890-000 Seropédica, Rio de Janeiro, Brasil. \\ 2 Departamento de Morfologia, Universidade Federal Fluminense. 24210-130 Niterói, Rio de Janeiro, Brasil. \\ E-mail: jurabreu@vm.uff.br \\ 3 Setor de Anatomia, Universidade Estácio de Sá. 22783-325 Rio de Janeiro, Brasil.
}

\begin{abstract}
Morphological characteristics of the cerebral vascular distribution of Sus scrofa Linnaeus (Mammalia, Artiodactyla). The aim this study was to verify the arterial vascularization of the wild boar brain, Sus scrofa Linnaeus, 1758, Suidae, and compare with other species. Five male and five female were injected with colored latex. The arterial supply of the brain was described and were done morphological analyses. In this species the arterial circuit of the brain is formed by the internal carotid artery branches, such as: the caudal communicant arteries, rostral branch, rostral cerebral arteries and rostral communicant arteries.
\end{abstract}

KEY WORDS. Arteries, basilar artery, circle of Willis, internal carotid artery.

RESUMO. O objetivo do presente estudo foi verificar a vascularização arterial do encéfalo do javali, Sus scrofa Linnaeus, 1758, Suidae, e comparar com outras espécies. Cinco machos e cinco fêmeas foram injetados com látex colorido. O suprimento arterial do encéfalo foi descrito e análises morfológicas foram feitas. Nesta espécie o circuito arterial do encéfalo é formado por ramos da artéria carótida interna, como: as artérias comunicantes caudais, o ramo rostral, as artérias cerebrais rostrais, e artérias comunicantes rostrais.

PALAVRAS CHAVE. Artérias, artéria basilar, artéria carótida interna, circulo de Willis.

O javali, Sus scrofa Linnaeus, 1758, Suidae, é um animal silvestre, ancestral do porco doméstico, e não pertence à fauna brasileira. No início do século XX, algumas amostras foram trazidos da Europa para a Argentina, com o objetivo de serem soltos em um parque de caça. Neste local, foram feitos algumas experiências, entre elas o cruzamento com o porco doméstico, para aumentar a prolificidade do javali puro que é baixa. Como resultado, a sua população escapou do controle e tornou-se hoje uma praga agrícola, não só na Argentina como também no Uruguai, onde invade plantações de milho, atacando e matando animais domésticos, como cachorros e filhotes de carneiro, (Nogueira-Filho 1999).

A morfologia dos animais silvestres exóticos é carente de informações básicas, especialmente quando se trata de espécies que apresentam algum potencial de exploração intensiva, seja como fonte de proteínas, ou modelo biológico, (Menezes et al. 2001), como é o caso do javali, criado sob controle do Iвама (Instituto Brasileiro de Meio Ambiente).

Esse animal é ancestral do porco doméstico e ambos são considerados da mesma espécie, uma vez que o cruzamento dos dois produz descendentes férteis. O porco doméstico nada mais é do que a conseqüência de processos de seleção e cruzamentos de diferentes variedades de javali, processo este iniciado na China por volta do ano 4.900 a.C, (Nogueira-Filho 1998, Nogueira-Filho \& Nogueira 2000).

Relativamente a estes mamíferos, as informações bibliográficas são escassas, quase sempre de caráter genérico, e poucas vezes oferecem dados específicos, como no caso do sistema arterial do encéfalo. A irrigação arterial das estruturas encefálicas de suínos é realizada de forma predominantemente pela artéria carótida interna (ACI) e artéria basilar (AB). A ACI é um ramo da artéria carótida comum (ACC), originada do tronco bi carotídeo, que se origina do tronco braquiocefálico, a artéria basilar é formada pela união das artérias vertebrais na face ventral da junção medulo-encefálica, (GETTy 1986).

O peso do encéfalo no homem representa $2 \%$ do peso corporal, no entanto usa $15 \%$ do débito cardíaco total e consome $20 \%$ da quantidade total disponível de oxigênio (Young $\&$ Young 1998). O sistema nervoso, como um todo, tem sido usado em estudos comparativos de desenvolvimento estrutural, variações anatômicas e dos arranjos vasculares, bem como de modelo cirúrgico (Ferreira \& Prada 2000). 
Existe uma carência de trabalhos sobre animais silvestres, principalmente da vascularização do encéfalo, esse é o caso do javali. O conhecimento da vascularização arterial do encéfalo do referido animal pode servir como subsídio à comparação com outros grupos de mamíferos. Portanto, o objetivo do trabalho foi descrever a morfologia da vascularização arterial do encéfalo do javali.

\section{MATERIAL E MÉTODOS}

Utilizou-se cabeças de animais, cinco machos e cinco fêmeas, oriundos do abatedouro "Pro-Fauna Ltda" localizado na cidade de Iguape São Paulo, que possui licença para abate e comercialização de animais silvestres, sob fiscalização do Serviço de Inspeção Federal (SIF) do IBAMA (Instituto Brasileiro de Meio Ambiente).

O sacrifício dos animais foi realizado de acordo com as normas do matadouro, sendo dessensibilizados por eletrochoque, sangrados e suspensos pelos apêndices pélvicos. Após secção das cabeças, foi realizado dissecção da artéria carótida comum de ambos os lados, ao nível do terço proximal do pescoço. Em seguida introduziu-se um cateter nas artérias e injetado água oxigenada a 10 volumes, por uma das artérias, para evitar a formação de coágulos e lavagem com água destilada, logo depois foi realizada a injeção do látex corado com pigmento vermelho (xadrez), até obter o retorno pela artéria adjacente, após então os vasos foram fechados com auxílio de fio de algodão. Em todos os animais retirou-se as calotas cranianas e realizado a incisão das meninges, após este procedimento, mergulhou-se as cabeças em solução de formol a $20 \%$ por cinco dias para fixação. Após fixação dos encéfalos, procedeu-se à retirada dos encéfalos da caixa craniana e posterior dissecção dos ramos das artérias carótidas internas intracraniais e basilar, bem como, mapeamento das ramificações para o encéfalo.

\section{RESULTADOS}

\section{Artéria carótida interna}

São originadas em ambos os lados das artérias carótidas comuns. São vasos pares que ascendem pelo canal carotídeo na base do encéfalo, perfuram a dura-máter, dão origem aos ramos comunicantes caudais, continuam rostralmente, passam ventralmente ao trato óptico, direcionam-se para o plano mediano dorsal ao nervo óptico e caudal ao tubérculo olfatório emitem as artérias cerebrais médias (um a três ramos)e continuam rostralmente como artérias cerebrais rostrais (Fig.1).

\section{Artéria cerebral média}

Possui de um a três segmentos, que surgem do ramo rostral da artéria carótida interna, percorrendo dorsolateralmente, rostral ao lobo piriforme e a superfície ventral da substância perfurada rostral, atingindo o sulco rinal lateral, distribuindo-se dorso-lateral e rostro-lateral no hemisfério cerebral (Fig. 1).

\section{Artéria cerebral rostral}

É uma continuação rostral da artéria carótida interna após emitir as artérias cerebrais médias, margeando a fissura longitudinal, após sua origem emite de um a dois pequenos ramos, formando uma rede de vasos chamado artéria comunicante rostral, ainda margeando a fissura mediana emite lateralmente um número de um a quatro ramos corticais que seguem em direção à superfície lateral do trato olfatório lateral, para se distribuírem nesse trato e em partes do lobo frontal dos hemisférios cerebrais. Após a emergência dos ramos corticais, caudalmente ao bulbo olfatório, a artéria cerebral rostral emite a artéria etmoidal interna e entra na fissura mediana unindo-se a uma curta distância com a artéria contra lateral para forma a artéria comum do corpo caloso (Fig. 1).

\section{Artéria comunicante caudal}

Após emergir da artéria carótida interna segue caudalmente e ventral aos pedúnculos cerebrais e próximo ao corpo mamilar emite a artéria cerebral caudal que percorre a face lateral do pedúnculo cerebral, e continua sobre a superfície ventral do pedúnculo cerebral em direção ao plano mediano onde emite a artéria do teto do mesencéfalo e continua para se anastomosar com a artéria contra lateral e basilar (Fig. 2).

\section{Artéria basilar}

É formada após anastomose das artérias vertebrais na altura do forame magno próximo a emergência do primeiro par de nervo cervical espinhal, percorre pela fissura mediana ventral e pelo sulco basilar na ponte até realizarem anastomose com as artérias comunicantes caudais na transição ponte mesencéfalo. No bulbo emite variáveis ramos bulbares pequenos e caudal na altura do corpo trapezóide emite as artérias cerebelares caudais, continuando seu trajeto, na ponte emite cerca de três a quatro artérias pontinas, na emergência do nervo trigêmeo emite a artéria cerebelar média e antes de sua anastomose com as artérias comunicantes caudais, emite $76,92 \%$ das artérias cerebelares rostrais direita, pois 23,08\% é emitido no ponto de anastomose com a artéria comunicante caudal, já as artérias cerebelares rostrais esquerda 69,23\% são emitido pela artéria basilar e $23,07 \%$ do ponto de anastomose entre a artéria basilar e a artéria comunicante caudal e; 7,7\%, sendo ramo direto da artéria comunicante caudal (Fig. 2).

Circuito arterial do cérebro. É constituído através dos ramos das artérias carótidas internas, tais como: artérias comunicantes caudais, ramos rostrais, cerebrais rostrais e comunicantes rostrais, na base do encéfalo, delimitando estruturas importantes, podendo citar a hipófise na porção central o quiasma óptico na porção rostral e o corpo mamilar na porção caudal do circuito.

Em todos encéfalos analisados observou-se uma forma regular dos arranjos das artérias que participam da formação do circuito arterial do cérebro, e subdividido em duas porções a partir da emergência da artéria carótida interna na base do encéfalo, uma rostral e a outra caudal (Fig. 2). 

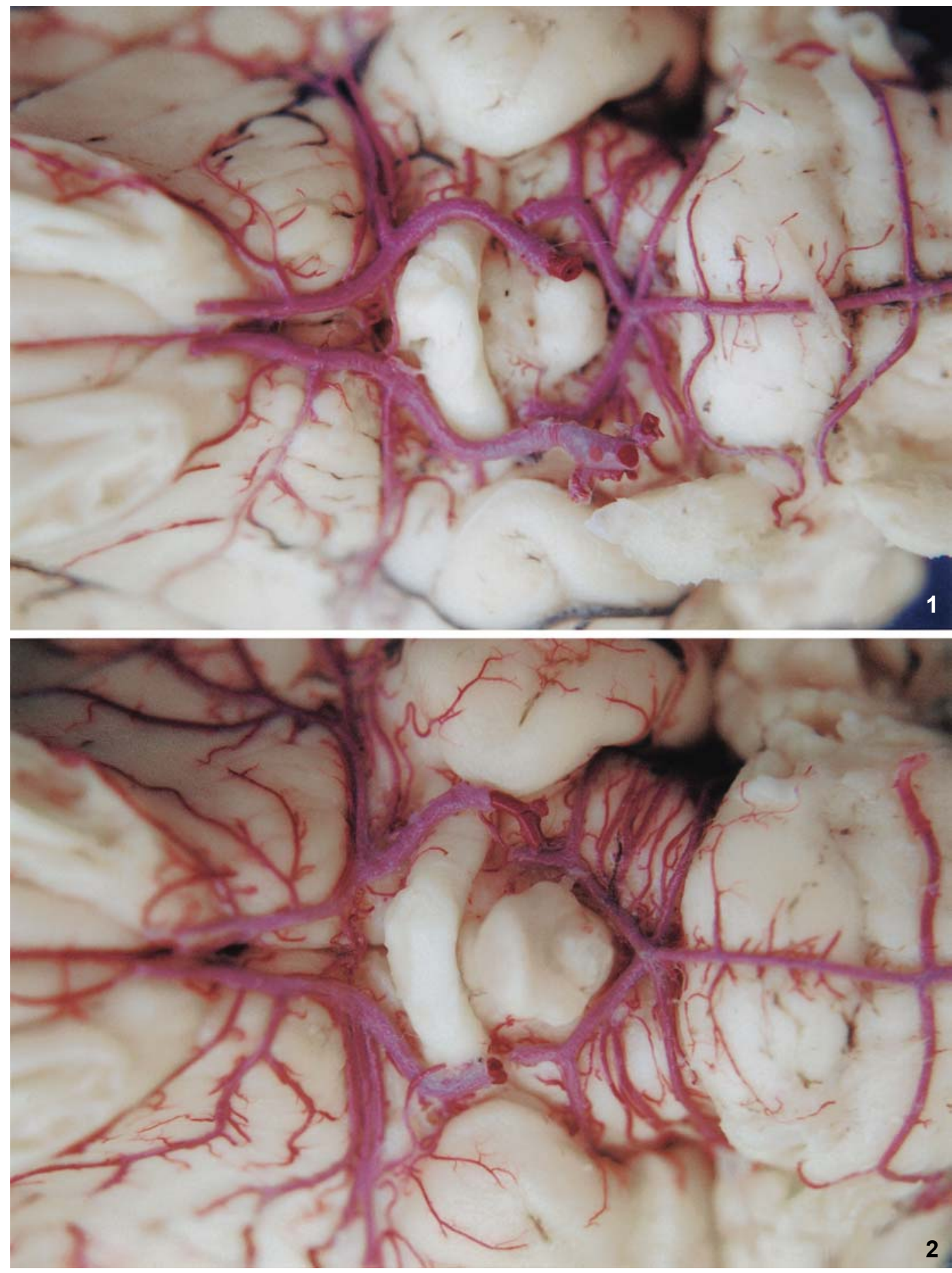

Figuras 1-2. Face ventral do encéfalo. Figura 1: artéria carótida interna (ACl), artéria comunicante caudal (1), ramo rostral (2), artéria cerebral média (3), artéria cerebral rostral (4), trato óptico (asterisco), (5) tubérculo olfatório (seta azul grande), ramo cortical; Figura 2: artéria comunicante caudal (1), artéria cerebral caudal (2), artéria do teto do mesencéfalo (3), anastomose das artérias comunicantes caudais com a artéria basilar (seta marela). (6), Artéria basilar (seta preta), artéria cerebelar Rostral (7), artéria cerebelar média (8), artéria cerebelar caudal (3), ramos pontinos (*), corpo mamilar (4) quiasma óptico (5). 


\section{DISCUSSÃO}

As artérias carótidas internas e basilar, com suas anastomoses estão localizadas na base do encéfalo, o arranjo formado por essas anastomoses é diferente entre as espécies domésticas, porém possui denominações diversas, no qual tornam complexos os estudos morfológicos.

SCHALler (1999), descreve as artérias da base do encéfalo dos suínos formando um círculo, denominado o mesmo de Círculo arterioso do cérebro ou de Willis. DE VRIESE (1905) ao realizar estudos da vascularização do encéfalo refere-se ao arranjo formado, como Polígono de Willis, uma figura geométrica na base do encéfalo. DE LA TORre et al. (1962), utilizam o termo círculo de Willis para as anastomoses arteriais na base do encéfalo. Alcântara \& Prada (1996a,b), em seus estudos utilizaram o termo circuito arterial do cérebro. De acordo com as observações no presente trabalho, as artérias da base do encéfalo não delimitam nenhuma forma geométrica correspondente a um polígono nem a um círculo. Estes resultados corroboram com AlCÂNTARA \& Prada (1996a,b), onde propõem uma revisão da Schaller (1999), afirmando que a formação arterial da base do encéfalo do cão não corresponde nem a polígono ou a círculo, mais sim a um circuito arterial. Sendo o termo circuito definido como uma linha que limita qualquer área fechada e a palavra círculo correspondente a uma região formando uma circunferência. Uma vez que, não foi observado nenhuma forma circular ou de polígono, este trabalho utilizou o termo circuito arterial do cérebro e está de acordo com (Alcântara \& Prada 1996b).

DE LA TORRE et al (1959) descrevem a vascularização do encéfalo do cão sendo proveniente das artérias carótidas interna e vertebrais. A artéria carótida interna subdividi-se em um ramo anterior e outro posterior, sendo o último a artéria comunicante posterior com o mesmo calibre da artéria carótida interna. O ramo anterior bifurca-se em artéria cerebral média e artéria cerebral anterior. O que não está de acordo com Alcântara \& Prada (1996a), onde relata que a artéria carótida interna bifurca-se em um ramo rostral, que ramifica-se em artéria cerebral media e cerebral rostral e o ramo caudal que dirige-se caudalmente e realiza anastomose com a artéria basilar.

GetTy (1986) cita que nos suínos domésticos a artéria carótida interna subdividi-se em ramos: um rostral que dividese em artéria cerebral média e artéria cerebral rostral e; um ramo caudal que é chamado de artéria comunicante caudal, que pode ser dividida em duas porções, uma proximal e outra distal chamada de artéria mesencefálica, a partir da emissão da artéria cerebral caudal. É denominada de comunicante, pois vai realizar anastomose com a artéria basilar. Porém neste estudo observou-se a artéria carótida interna com dois ramos: um rostral, que ramifica em artéria cerebral média e artéria cerebral rostral; outro caudal que chamamos de artéria comunicante caudal que irá ramificar-se durante seu trajeto, onde o principal ramo é a artéria cerebral caudal. Estas observações corroboram com as de AlcÂntara \& Prada (1996a) em relação a nomenclatura utilizada para ramificação da artéria carótida interna rostralmente, porém discorda quando o mesmo nomeia a bifurcação caudal da artéria carótida interna em ramo caudal, e concorda com DE LA TORRe et al. (1959) quando nomeia em ramo caudal da artéria carótida interna em artéria comunicante caudal. As observações realizadas neste estudo são completamente contrárias as realizadas por NANDA (1986) que cita a artéria carótida interna com três ramos, as artérias comunicantes caudal e rostral e artéria cerebral média.

No presente estudo foi observado originando do ramo rostral da artéria carótida interna, de uma a três artérias cerebrais médias e artéria cerebral rostral, este dados também são encontrados em (GeTTY 1986).

Segundo DE LA TORRE et al. (1959) em cães as artérias vertebrais surgem das artérias subclávias e ascendem no canal transverso pelos forames transversos. As artérias vertebrais se unem para formar a artéria basilar. Esta última, freqüentemente, tem um curso sinuoso na face ventral do tronco encefálico; dividese no ponto mais alto da ponte para formar a porção posterior do círculo de Willis. A artéria basilar cursa ventralmente para o tronco cerebral sinuosamente, bifurcando-se nas duas artérias comunicantes posteriores. Os maiores ramos das artérias comunicantes posteriores são as artérias cerebelares superiores e as cerebrais posteriores. GetTy (1986) cita que em suínos as artérias cerebelares rostrais é ramo da artéria comunicante caudal, e de acordo com as observações feitas neste trabalho em relação à artéria basilar do javali podemos afirmar que, a artéria basilar é formada a partir da anastomose das artérias vertebrais e emiti diversos ramos, onde os principais são as artérias cerebelares caudal, média e sendo a artéria cerebelar rostral direita emitida $76,92 \%$ e $23,08 \%$ é emitido no ponto de anastomose com a artéria comunicante caudal, enquanto a esquerda $69,23 \%$ é emitido pela basilar e $23,07 \%$ do ponto de anastomose com a artéria comunicante caudal e 7,7\% sendo ramo da artéria comunicante caudal, contra dizendo os autores acima, quando ele citam que a artéria cerebelar rostral é predominantemente ramo da artéria comunicante caudal.

Segundo GilliLAn (1976) em mamíferos primitivos a artéria carótida interna é ainda a maior fonte de suprimento sanguíneo para o cérebro, mais as artérias vertebrais contribuem para o suprimento do tronco encefálico. O padrão das artérias que suprem o encéfalo de mamíferos superiores é variável e complexo. Nos suínos domésticos o encéfalo recebe suprimento sanguíneo das artérias carótida interna e basilar, no qual concorda-se, pois foi observado em nossos resultados as duas artérias contribuindo para vascularização arterial do encéfalo, sendo a artéria basilar responsável por nutrir o tronco encefálico e cerebelo e as artérias carótidas internas o cérebro, que nos faz discordar de Goss (1988) que descreve uma parte considerável do cérebro sendo irrigado pelas duas artérias vertebrais, através da artéria basilar.

\section{REFERÊNCIAS BIBLIOGRÁFICAS}

AlCÂNTARa, M.A.; I.L.S. Prada. 1996a. Arteries of basis of encephalon in dog (Canis familiaris Linnaêus, 1758). I. Anatomical

Revista Brasileira de Zoologia 21 (4): 955-959, dezembro 2004 
study od sources and behaviour. Brazilian Journal of Veterinary Research and Animal Science, São Paulo, 33 (2): 67-71.

.1996b. Arteries of basis of encephalon in dog (Canis familiaris, linnaeus, 1758). II. Formation and behaviour of the encephalon arterial circuit. Brazilian Journal of Veterinary Research and Animal Science, São Paulo, 33 (2): 72-76.

de la Torre, E.; M.G. Netsky \& I. Meschan. 1959. Intracranial e extracranial circulation in the dog: anatomic and angiografic studies. American Journal of Anatomy, Philadelphia, 105: 343-381.

de la Torre, E.; O.C. Mitchell \& M.G. Netsky. 1962. Anatomic and angiographic studies of the vertebral basilar system in dog. American Journal of Anatomy, Philadelphia, 110: 187-198.

DE VRIESE, B. 1905. Sur la signification morphologique des artères cerebrales. Archives de Biologie, Paris, 21: 357-457.

Ferreira, C.G.; I.L.S. Prada. 2000. Estudo anatômico das artérias da base do encéfalo de suínos (Sus scrofa domésticos Linnaeus, 1758). Brazilian Journal Morphological Sciences, São Paulo, 17: 239-240.

Getty, R. 1986. Anatomia dos Animais Domésticos. Rio de Janeiro, Guanabara Koogan, vol. 2, 5a ed., 2000p.

GILLILAN, L.A. 1976. Extra and intra-cranial blood supply to brain of dog and cat. American Journal of Anatomy, Philadelphia, 146: 237-254.

Goss, C.M. 1988. Anatomia. Rio de Janeiro, Guanabara Koogan, $29^{a}$ ed., 1147 p.

Menezes, D.A.; M.A.M. Carvalho; M.F. Cavalcante Filho \& W.M. SouzA. 2001. Configuração do sistema venoso portal na cutia (Dasyprocta aguti, Rodentia) Brazilian Journal of Veterinary Research and Animal Science, São Paulo, 38 (6): 263-266.

NANDA, B.S. 1986. Suprimento sanguíneo para o cérebro, p. 15131518. In: R. GetTy, S. Sisson \& J.D. Grossman (Eds). Anatomia dos Animais Doméstico. Rio de Janeiro, Guanabara Koogan, $6^{\mathrm{a}}$ ed., 1788p.

Nogueira-Filho, S.L.G.1998. Manual de criação de javali. Viçosa, Centro de Produções Técnicas, 50p.

- 1999. Manual de Criação de Cateto e Queixada. Viçosa, Centro de Produções Técnicas, vol.1, 50p.

Nogueira-Filho, S.L.G.; S.S.C. Nogueira. 2000. Criação comercial de animais silvestres: produção e comercialização da carne e subprodutos na região sudeste do Brasil. Revista econômica do nordeste, Fortaleza, 1 (2): 1-50.

SCHALLER, O. 1999. Nomenclatura Anatômica veterinária. São Paulo, Manole, $1^{\mathrm{a}}$ ed., 614p.

Young, P.A. \& P.H. Young. 1998. Bases da neuroanatomia clínica. Rio de Janeiro, Editora Guanabara Koogan, 285p.

Recebido em 08.I.2004; aceito em 19.XI.2004. 